\title{
ANALYSIS OF THE BOUNDARY VALUE PROBLEM ASSOCIATED WITH THE NONRELATIVISTIC THOMAS-FERMI EQUATION FOR HEAVY ATOMS IN INTENSE MAGNETIC FIELDS
}

\author{
Christopher C. Tisdell And MARK Holzer
}

\begin{abstract}
This article presents a firm mathematical foundation for the boundary value problem associated with the nonrelativistic Thomas-Fermi equation for heavy atoms in intense magnetic fields. Our approach uses an application of differential inequalities and ideas from nonlinear analysis, including: the technique of lower and upper solutions; and fixed-point theory. We present new results that ensure existence, uniqueness, location and approximation of solutions. We thus establish that the Thomas-Fermi model leads to a robust theory of heavy atoms in intense magnetic fields in spite of the severe approximations that it employs.

A YouTube video from the first author that is designed to complement this paper can be found here http://tinyurl.com/ThomasFermi.
\end{abstract}

\section{Mathematics subject classification (2010): 34B60, 34B15.}

Keywords and phrases: Thomas-Fermi equation, boundary value problem, heavy atoms in intense magnetic fields, qualitative analysis of solutions, existence, uniqueness and location of solutions.

\section{REFERENCES}

[1] R.P. AgARWAL, Boundary value problems for higher order differential equations. World Scientific, Singapore, 1986.

[2] E. AkKermans, S. Ghosh, Z.H Musslimani, Numerical study of one-dimensional and interacting Bose-Einstein condensates in a random potential, J. Phys. B: At. Mol. Opt. Phys., 41 (2008), no. 4, Article ID 045302 (12pp).

[3] D.R. Ballantyne, Reflection spectra from an accretion disc illuminated by a neutron star X-ray burst, Mon. Not. R. Astron. Soc., 351 (2004), 57-62.

[4] M.A. BAUTISTA, Electronic correlations and polarizability of the Thomas-Fermi-Dirac-Amaldi potential: applications to the singly ionized iron-peak species, J. Phys. B: At. Mol. Opt. Phys. 41 (2008), no. 6, Article ID 065701 (8pp).

[5] Ph. BÉnilan, H. Brézis, Nonlinear problems related to the Thomas-Fermi equation. Dedicated to Philippe Bénilan, J. Evol. Equ., 3 (2003), no. 4, 673-770.

[6] R. Benguria, H. Brézis, E.H. Lieb, The Thomas-Fermi-von Weizsäcker theory of atoms and molecules, Comm. Math. Phys., 79 (1981), no. 2, 167-180.

[7] R. Benguria, E.H. Lieb, Many-body atomic potentials in Thomas-Fermi theory, Ann. Phys. NY 110, (1978) 34-45.

[8] R. Benguria, E.H. Lieb, The positivity of the pressure in Thomas-Fermi theory, Comm. Math. Phys., 63 (1978), 193-218.

[9] R.K. Bhaduri, V.M.N. Murthy, M. Brack, Fermionic ground state at unitarity and Haldane exclusion statistics, J. Phys. B: At. Mol. Opt. Phys., 41 (2008), no. 11, Article ID 115301 (4pp).

[10] H. BRÉZIS, Nonlinear problems related to the Thomas-Fermi equation. Contemporary developments in continuum mechanics and partial differential equations (Proc. Internat. Sympos., Inst. Mat., Univ. Fed. Rio de Janeiro, Rio de Janeiro, 1977), pp. 81-89, North-Holland Math. Stud., 30, North-Holland, Amsterdam-New York, 1978. 
[11] H. BRÉZIS, Nonlinear equations of Thomas Fermi type. Proceedings of the First Franco-Southeast Asian Mathematical Conference (Nanyang Univ., Singapore, 1979). Southeast Asian Bull. Math. 1979, Special Issue, 167-171.

[12] H. BRÉZIS, Some variational problems of the Thomas-Fermi type. Variational inequalities and complementarity problems (Proc. Internat. School, Erice, 1978), pp. 53-73, Wiley, Chichester, 1980.

[13] H. BRÉZIS, A free boundary problem in quantum mechanics: Thomas-Fermi equation. Free boundary problems, Vol. II (Pavia, 1979), pp. 85-91, Ist. Naz. Alta Mat. Francesco Severi, Rome, 1980.

[14] H. BrÉZIS, E.H. LIEB, Long range atomic potentials in Thomas-Fermi theory, Comm. Math. Phys., 65 (1979), no. 3, 231-246.

[15] M.A. Caracanhas, J.A. Seman, E.R.F. Ramos, E.A.L. Henn, K.M.F. Magalhaes, K. Helmerson, V.S. BAGNato, Finite temperature correction to the Thomas-Fermi approximation for a Bose-Einstein condensate: comparison between theory and experiment, J. Phys. B: At. Mol. Opt. Phys., 42 (2009), no. 14, Article ID 145304, (8pp).

[16] C. De CosteR, P. HABETS, The lower and upper solutions method for boundary value problems. Handbook of differential equations, 69-160, Elsevier North-Holland, Amsterdam, 2004.

[17] P.A.M. DiRAC, Note on the exchange phenomena in the Thomas atom, Proc. Cambridge Philos. Soc., 26 (1930), 376.

[18] E. FeRMi, Un metodo statistico per la determinazione di alcune priorietà dell'atome, Rend. Addad. Naz. Lincei 6 (1927), 602.

[19] M.D. Glossman, E.A. CAStro, Approximate analytical solution of the Thomas-Fermi equation for positive ions in a strong magnetic field, Z. Phys. D - Atoms, Molecules and Clusters, 6 (1987), $81-82$.

[20] A. Granas, R.B. Guenther, J.W. Lee, A note on the Thomas-Fermi equation, Z. Angew. Math. Mech., 61 (1981), no. 3, 204-205.

[21] M. GUPTA, K.R. KRISHNA, An analytical approach for the nonlinear modified Thomas-Fermi equation to derive the ground-state and dynamic properties of a spherically and cylindrically trapped Bose-Einstein condensate, J. Phys. B: At. Mol. Opt. Phys., 41 (2008), no. 19, Article ID 195302, (8pp).

[22] P. Hartman, Ordinary differential equations. Corrected reprint of the second (1982) edition. With a foreword by Peter Bates. Classics in Applied Mathematics, 38. Society for Industrial and Applied Mathematics (SIAM), Philadelphia, PA, 2002.

[23] S.H. Hill, P.J. Grout, N.H. MarCh, Relativistic Thomas-Fermi equation in an extremely high magnetic field, and its solution for atomic ions, J. Phys. B: At. Mol. Phys., 18 (1985), 4665-4673.

[24] S.H. HiLl, P.J. Grout, N.H. MARCH, Chemical potential and total energy of heavy positive ions in extremely strong magnetic fields, near the weak ionisation limit, J. Phys. B: At. Mol. Phys., 16 (1983), 2301-2307.

[25] E. Hille, On the Thomas-Fermi equation, Proc. Nat. Acad. Sci. U.S.A., 62 (1969), 7-10.

[26] A.I. Ibrahim, S. Safi-Harb, J.H. Swank, W. Parke, S. Zane, R. Turolla, Discovery of cyclotron resonance features in the soft gamma repeater SGR 1806-20. The Astrophysical Journal, 574:L51-L55, (2002).

[27] B.B Kadomstev, Heavy atom in an ultrastrong magnetic field, Soviet Physics JETP, 31 (1970), no. $5,945-947$.

[28] A.S. Kornev, B.A. Zon, Rydberg spectra of atoms and positive ions in the Thomas-Fermi model, J. Phys. B: At. Mol. Opt. Phys., 36 (2003), no. 19, 4027-4034.

[29] E.H. Lieb, J.P. Solovej, J. YngVason, Asymptotics of Heavy atoms in High magnetic Fields: I. Lowest Landau Band Regions, Commun. Pure. Appl. Math., 47 (1994), 513-591.

[30] E.H. Lieb, J.P. Solovej, J. Yngvason, Asymptotics of Heavy Atoms in High Magnetic Fields: II. Semiclassical Regions, Commun. Math. Phys., 161 (1994), 77-124.

[31] E.H. Lieb, Thomas-Fermi and Hartree-Fock theory. Proceedings of the International Congress of Mathematicians (Vancouver, B. C., 1974), Vol. 2, pp. 383-386. Canad. Math. Congress, Montreal, Que., 1975.

[32] E.H. LiEB, The stability of matter, Rev. Mod. Phys., 48 (1976), 553-569.

[33] E.H. Lieb, B. Simon, Thomas-Fermi theory revisited, Phys. Rev. Lett., 31 (1973), 681-683.

[34] E.H. Lieb, B. Simon, The Thomas-Fermi theory of atoms, molecules and solids, Adv. Math., 23, (1977), 22-116. 
[35] R.O. Mueller, A.R.P. Rau, L. Spruch, Statistical Model of Atoms in Intense Magnetic Fields, Phys. Rev. Lett., 26 (1971), 1136-1139.

[36] E. TELLER, On the stability of molecules in the Thomas-Fermi theory, Rev. Mod. Phys., 34 (1962), $627-631$.

[37] A.Z. Msezane, Z. Felfli, D. Sokolovs Ki, Near-threshold resonances in electron elastic scattering cross sections for Au and Pt atoms: identification of electron affinities, J. Phys. B: At. Mol. Opt. Phys., 41 (2008), no. 10, Article ID 105201, (6pp).

[38] D. O’Regan, Theory of Singular boundary Value Problems. World Scientific Publishing Co., Inc., River Edge, NJ, 1994.

[39] A.L.A. Penna, J.B. Diniz, F.A. Oliveria, Charge degeneracy removal in the screened hydrogen atom, J. Phys. B: At. Mol. Opt. Phys., 41 (2008), no. 19, Article ID 195302, (8pp).

[40] M.H. Protter, H.F. Weinberger, Maximum principles in differential equations. Corrected reprint of the 1967 original. Springer-Verlag, New York, 1984.

[41] L.H. Thomas, The calculation of atomic fields, Proc. Cambridge Phil. Soc., 23 (1927), 542.

[42] C. Tisdell, What is a Lipschitz condition? Available on Dr Chris Tisdell's YouTube Channel, 14/02/2014, Accessed on 04/11/2014 http: //www . youtube. com/watch?v=Cnc83B3C2pY

[43] C. TISDELL, Banach fixed point theorem and differential equations. Available on Dr Chris Tisdell's YouTube Channel, 08/09/2014, Accessed on 04/11/2014 http: //www . youtube. com/watch?v=RhAjh8QpkDY

[44] E. ZEIDlER, Nonlinear functional analysis and its applications. I. Fixed-point theorems. Translated from the German by Peter R. Wadsack. Springer-Verlag, New York, 1986. 